\title{
EXISTENCE OF EXTREMAL PERIODIC SOLUTIONS FOR QUASILINEAR PARABOLIC EQUATIONS
}

\author{
SIEGFRIED CARL
}

\begin{abstract}
In this paper we consider a quasilinear parabolic equation in a bounded domain under periodic Dirichlet boundary conditions. Our main goal is to prove the existence of extremal solutions among all solutions lying in a sector formed by appropriately defined upper and lower solutions. The main tools used in the proof of our result are recently obtained abstract results on nonlinear evolution equations, comparison and truncation techniques and suitably constructed special testfunction.
\end{abstract}

\section{INTRODUCTION}

Let $\Omega \subset \mathbb{R}^{N}$ be a bounded domain with Lipschitz boundary $\partial \Omega, Q=$ $\Omega \times(0, \tau)$ and $\Gamma=\partial \Omega \times(0, \tau), \tau>0$. This paper deals with weak solutions of the following quasilinear Dirichlet-periodic boundary value problem (PBVP for short)

$$
\left.\begin{array}{r}
\frac{\partial u}{\partial t}+A u=f(x, t, u, \nabla u) \text { in } Q, \\
u(x, 0)=u(x, \tau) \text { in } \Omega \text { and } u=0 \text { on } \Gamma,
\end{array}\right\}
$$

where $A$ is a second order quasilinear differential operator in divergence form of Leray-Lions type given by

$$
A u(x, t)=-\sum_{i=1}^{N} \frac{\partial}{\partial x_{i}} a_{i}(x, t, u(x, t), \nabla u(x, t)), \text { and } \nabla u=\left(\frac{\partial u}{\partial x_{1}}, \cdots, \frac{\partial u}{\partial x_{N}}\right) .
$$

1991 Mathematics Subject Classification. 35B05, 35B10, 35K60, 47N20.

Key words and phrases. Quasilinear parabolic equations, Dirichlet-periodic boundary conditions, extremal solutions, upper and lower solutions, pseudomonotone operators, truncation and comparison techniques.

Received: August 18, 1997. 
Assuming the existence of bounded upper and lower solutions an existence result for problem (1.1) has been proved in a paper by Deuel and Hess in [7] by applying the penalty method to an appropriately associated auxiliary parabolic variational inequality.

The main goal of the present paper is to extend this result by proving the existence of extremal periodic solutions among all the solutions of the PBVP (1.1) within the sector formed by not necessarily bounded upper and lower solutions. The proof of this extremality result is done by showing that the solution set $\mathcal{S}$ enclosed by the upper and lower solutions possesses the properties of directedness and of inductivity, where the latter means that any well-ordered chain in $\mathcal{S}$ has the least upper bound in $\mathcal{S}$. This, however, requires a method of proof that is essentially different from that used in [7].

The corresponding stationary problem to (1.1) has been treated in different ways by Puel [11] and the author [4]. The technique used by Puel to treat the associated elliptic problem is based among others on the lattice structure of the underlying solution space which is the Sobolev space $W_{0}^{1, p}(\Omega)$. However, in the parabolic case considered here the underlying solution space of problem (1.1) will be the Lions space $\mathcal{W}$ which is defined by

$$
\mathcal{W}:=\left\{u \in \mathcal{V}:=L^{p}\left(0, \tau ; W_{0}^{1, p}(\Omega)\right) \mid \frac{\partial u}{\partial t} \in \mathcal{V}^{*}\right\},
$$

where $\mathcal{V}^{*}$ denotes the dual space to $\mathcal{V}$. Due to the lack of regularity of the time derivative the space $\mathcal{W}$, in general, does not possess lattice stucture, and thus the extension of the extremal solution result for elliptic problems according to [11] to the general quasilinear parabolic problem (1.1) considered here is by no means straightforward and requires completely different tools. Only recently in a paper by Grenon [8] (cf. also [9]) the existence of extremal solutions for quasilinear parabolic equations under initial and Dirichlet boundary conditions has been considered. In [8] the method of proof is based on regularization techniques and follows an idea used by Puel in the elliptic case. Moreover, in Grenon's paper the coefficients $a_{i}=a_{i}(x, t, s, \xi)$ of the operator $A$ are assumed to satisfy a Lipschitz condition with respect to the variable $s$ standing for the solution $u$.

In this paper we provide an alternative approach to prove extremality results which at the same time allows to treat a more general dependence of the coefficients $a_{i}$ on the variable $s$ expressed in terms of a modulus of continuity condition. The interdependence of various types of monotonicity conditions of the operator $A$ and the modulus of continuity condition of the coefficients $a_{i}$ with respect to $s$ is discussed. Our approach is mainly based on an associated auxiliary problem that arises from the original one by truncation procedures and on special test function techniques. The main tools used in the proof are existence results for nonlinear evolution equations developed recently in [1] and comparison techniques.

The method of proof given here is a strong generalization of the method developed in a recent paper by the author in [3] where initial and Dirichlet 
boundary conditions and an operator $A$ of the form

$$
A u(x, t)=-\sum_{i=1}^{N} \frac{\partial}{\partial x_{i}} a_{i}(x, t, \nabla u(x, t)),
$$

whose coefficients $a_{i}$ do not depend on $s$ have been taken into account.

Finally it should be noted that the results of this paper hold true also in case of initial-Dirichlet boundary conditions.

\section{Hypotheses, Definitions AND the MAIN RESUlt}

Let $W^{1, p}(\Omega)$ denote the usual Sobolev space and $\left(W^{1, p}(\Omega)\right)^{*}$ its dual space. For the sake of simplicity we shall assume $p \geq 2$, and $q \in \mathbb{R}$ being the dual real satisfying $1 / p+1 / q=1$. Then $W^{1, p}(\Omega) \subset L^{2}(\Omega) \subset\left(W^{1, p}(\Omega)\right)^{*}$ forms an evolution triple with all the embeddings being continuous, dense and compact, cf. [12].

We set $\mathcal{V}=L^{p}\left(0, \tau ; W^{1, p}(\Omega)\right)$, denote its dual space by $\mathcal{V}^{*}=L^{q}\left(0, \tau ;\left(W^{1, p}(\Omega)\right)^{*}\right)$, and define a function space $\mathcal{W}$ by

$$
\mathcal{W}=\left\{w \in \mathcal{V} \mid \frac{\partial w}{\partial t} \in \mathcal{V}^{*}\right\}
$$

where the derivative $\partial / \partial t$ is understood in the sense of vector-valued distributions, cf. [12]. The space $\mathcal{W}$ endowed with the norm

$$
\|w\|_{\mathcal{W}}=\|w\|_{\mathcal{V}}+\|\partial w / \partial t\|_{\mathcal{V}^{*}}
$$

is a Banach space which is separable and reflexive due to the separability and reflexivity of $\mathcal{V}$ and $\mathcal{V}^{*}$, respectively. Furthermore it is well known that the embedding $\mathcal{W} \subset C\left([0, \tau], L^{2}(\Omega)\right)$ is continuous, cf. [10, 12]. Finally, because $W^{1, p}(\Omega) \subset L^{p}(\Omega)$ is compactly embedded, we have a compact embedding of $\mathcal{W} \subset L^{p}(Q)$, cf. $[10,12]$.

By $W_{0}^{1, p}(\Omega)$ we denote the subspace of $W^{1, p}(\Omega)$ whose elements have generalized homogeneous boundary values. Let $W^{-1, q}(\Omega)$ denote the dual space of $W_{0}^{1, p}(\Omega)$. Then obviously $W_{0}^{1, p}(\Omega) \subset L^{2}(\Omega) \subset W^{-1, q}(\Omega)$ forms an evolution triple and all statements made above remain true also in this situation when setting $\mathcal{V}_{0}=L^{p}\left(0, \tau ; W_{0}^{1, p}(\Omega)\right), \mathcal{V}_{0}^{*}=L^{q}\left(0, \tau ; W^{-1, q}(\Omega)\right)$ and $\mathcal{W}_{0}=\left\{w \in \mathcal{V}_{0} \mid \frac{\partial w}{\partial t} \in \mathcal{V}_{0}^{*}\right\}$

We impose the following conditions of Leray-Lions type on the coefficient functions $a_{i}: Q \times \mathbb{R} \times \mathbb{R}^{N} \mapsto \mathbb{R}, i=1, \ldots, N$.

(A1) Each $a_{i}: Q \times \mathbb{R} \times \mathbb{R}^{N} \mapsto \mathbb{R}$ satisfies Carathéodory conditions, i.e., $a_{i}(x, t, s, \xi)$ is measurable in $(x, t) \in Q$ for all $(s, \xi) \in \mathbb{R} \times \mathbb{R}^{N}$ and continuous in $(s, \xi)$ for almost all $(x, t) \in Q$. There exist a constant $c_{0}>0$ and a function $k_{0} \in L^{q}(Q), 1 / p+1 / q=1$, such that

$$
\left|a_{i}(x, t, s, \xi)\right| \leq k_{0}(x, t)+c_{0}\left(|s|^{p-1}+|\xi|^{p-1}\right)
$$


$(\mathrm{A} 2)$

for a.e. $(x, t) \in Q$ and for all $(s, \xi) \in \mathbb{R} \times \mathbb{R}^{N}$.

$$
\sum_{i=1}^{N}\left(a_{i}(x, t, s, \xi)-a_{i}\left(x, t, s, \xi^{\prime}\right)\right)\left(\xi_{i}-\xi_{i}^{\prime}\right) \geq \mu\left|\xi-\xi^{\prime}\right|^{p}
$$

for a.e. $(x, t) \in Q$, for all $s \in \mathbb{R}$, and for all $\xi, \xi^{\prime} \in \mathbb{R}^{N}$ with $\mu$ being some positive constant.

(A3)

$$
\begin{aligned}
& \left|a_{i}(x, t, s, \xi)-a_{i}\left(x, t, s^{\prime}, \xi\right)\right| \\
& \leq\left[k_{1}(x, t)+|s|^{p-1}+\left|s^{\prime}\right|^{p-1}+|\xi|^{p-1}\right] \omega\left(\left|s-s^{\prime}\right|\right),
\end{aligned}
$$

for some function $k_{1} \in L^{q}(Q)$, for a.e. $(x, t) \in Q$, for all $s, s^{\prime} \in \mathbb{R}$ and for all $\xi \in \mathbb{R}^{N}$, where $\omega:[0, \infty) \mapsto[0, \infty)$ is the modulus of continuity satisfying

$$
\int_{0^{+}} \frac{d r}{\omega^{q}(r)}=+\infty,
$$

which means that for any $\varepsilon>0$ the integral taken over $[0, \varepsilon]$ is divergent, i.e., we have $\int_{0}^{\varepsilon} \frac{d r}{\omega^{q}(r)}=+\infty$.

Remark 2.1. The proof of our extremality result, in particular the proof of directedness of the solution set, requires a strong monotonicity condition (A2) which is related with the modulus of continuity condition (A3). There is an interplay between p-ellipticity and the q-modulus of continuity. $\mathrm{Hy}$ pothesis (A3) is satisfied for example in case that $\omega\left(\left|s-s^{\prime}\right|\right)=c\left|s-s^{\prime}\right|^{1 / q}$ with some positive constant $c$, i.e., the coefficients $a_{i}(x, t, s, \xi)$ satisfy a Hölder condition with respect to $s$. However, if we impose instead of (2.1) the more restrictive condition

$$
\int_{0^{+}} \frac{d r}{\omega(r)}=+\infty
$$

which includes for example $\omega\left(\left|s-s^{\prime}\right|\right)=c\left|s-s^{\prime}\right|$, i.e., a Lipschitz condition with respect to $s$ then one can relax the strong monotonicity condition (A2) by a strict monotonicity condition $\left(\mathrm{A} 2_{1}\right)$ and a coercivity condition $\left(\mathrm{A} 2_{2}\right)$, i.e.,

$\left(\mathrm{A} 2{ }_{1}\right)$

$$
\sum_{i=1}^{N}\left(a_{i}(x, t, s, \xi)-a_{i}\left(x, t, s, \xi^{\prime}\right)\right)\left(\xi_{i}-\xi_{i}^{\prime}\right)>0
$$



$\left(\mathrm{A} 2_{2}\right)$

for a.e. $(x, t) \in Q$, for all $s \in \mathbb{R}$, and for all $\xi, \xi^{\prime} \in \mathbb{R}^{N}$ with $\xi \neq \xi^{\prime}$.

$$
\sum_{i=1}^{N} a_{i}(x, t, s, \xi) \xi_{i} \geq \nu|\xi|^{p}-k(x, t)
$$

for a.e. $(x, t) \in Q$, for all $s \in \mathbb{R}$, and for all $\xi \in \mathbb{R}^{N}$ with some constant $\nu>0$ and some function $k \in L^{1}(Q)$. In particular (A2) may be replaced by the weaker conditions $\left(\mathrm{A} 2_{1}\right)$ and $\left(\mathrm{A} 2_{2}\right)$ if the coefficients $a_{i}$ do not depend on $s$.

Let us denote by $\langle\cdot, \cdot\rangle$ the duality pairing between the elements of $\mathcal{V}^{*}$ and $\mathcal{V}$ (respectively $\mathcal{V}_{0}^{*}$ and $\mathcal{V}_{0}$ ). Then as a consequence of (A1) and (A2) the semilinear form $a$ associated with the operator $A$ by

$$
\langle A u, \varphi\rangle=a(u, \varphi)=\sum_{i=1}^{N} \int_{Q} a_{i}(x, t, u, \nabla u) \frac{\partial \varphi}{\partial x_{i}} d x d t
$$

is well-defined on $\mathcal{V} \times \mathcal{V}$ and the operator $A: \mathcal{V} \mapsto \mathcal{V}^{*}$ (respectively $\mathcal{V}_{0} \mapsto \mathcal{V}_{0}^{*}$ ) is continuous and bounded. The norm (strong) convergence is denoted by $\rightarrow$, and the weak convergence by $\rightarrow$.

A partial ordering in $L^{p}(Q)$ is defined by $u \leq w$ if and only if $w-u$ belongs to the set $L_{+}^{p}(Q)$ of all nonnegative elements of $L^{p}(Q)$. This induces a corresponding partial ordering also in the subset $\mathcal{W}$ of $L^{p}(Q)$, and if $\underline{u}, \bar{u} \in$ $\mathcal{W}$ with $\underline{u} \leq \bar{u}$ then

$$
[\underline{u}, \bar{u}]=\{u \in \mathcal{W} \mid \underline{u} \leq u \leq \bar{u}\}
$$

denotes the order interval formed by $\underline{u}$ and $\bar{u}$. Further we assume that the function $f: Q \times \mathbb{R} \times \mathbb{R}^{N} \mapsto \mathbb{R}$ satisfies the Carathéodory conditions and associate with it its Nemytskij operator $F$ defined by

$$
F u(x, t)=f(x, t, u(x, t), \nabla u(x, t)) .
$$

Let us introduce the notion of a (weak) solution of the PBVP (1.1).

Definition 2.1. A function $u \in \mathcal{W}_{0}$ is called a solution of problem (1.1) if $F u \in L^{q}(Q)$ such that

(i) $u(\cdot, 0)=u(\cdot, \tau)$ in $\Omega$,

(ii) $\left\langle\frac{\partial u}{\partial t}, \varphi\right\rangle+a(u, \varphi)=\int_{Q} F u \varphi d x d t$, for all $\varphi \in \mathcal{V}_{0}$.

We define an upper solution for (1.1) as follows.

Definition 2.2. A function $\bar{u} \in \mathcal{W}$ is called an upper solution to PBVP (1.1) if $F \bar{u} \in L^{q}(Q)$ and

(i) $\bar{u} \geq 0 \quad$ on $\Gamma, \quad \bar{u}(\cdot, 0) \geq \bar{u}(\cdot, \tau)$ in $\Omega$,

(ii) $\left\langle\frac{\partial \bar{u}}{\partial t}, \varphi\right\rangle+a(\bar{u}, \varphi) \geq \int_{Q} F \bar{u} \varphi d x d t$, for all $\varphi \in \mathcal{V}_{0} \cap L_{+}^{p}(Q)$. 
Similarly a function $\underline{u} \in \mathcal{W}$ is a lower solution to (1.1) if the reversed inequalities hold in (i) and (ii) of Definition 2.2.

Further we shall make the following hypotheses.

(H1) Suppose PBVP (1.1) has an upper solution $\bar{u}$ and a lower solution $\underline{u}$ such that $\underline{u} \leq \bar{u}$.

(H2) There exist a function $k_{2} \in L_{+}^{q}(Q)$ and a constant $c_{1} \geq 0$ such that

$$
|f(x, t, s, \xi)| \leq k_{2}(x, t)+c_{1}|\xi|^{p-1}
$$

for a.e. $(x, t) \in Q$ and for all $\xi \in \mathbb{R}^{N}$ and for all $s \in[\underline{u}(x, t), \bar{u}(x, t)]$. A solution $u^{*}$ is the greatest solution within $[\underline{u}, \bar{u}]$ if for any solution $u \in[\underline{u}, \bar{u}]$ we have $u \leq u^{*}$. Similarly, $u_{*}$ is the least solution in $[\underline{u}, \bar{u}]$ if for any solution $u \in[\underline{u}, \bar{u}]$ it holds $u_{*} \leq u$. The least and greatest solutions are the extremal ones.

The main result of this paper is the following existence and extremality theorem.

Theorem 2.1. Let hypotheses (A1)-(A3) and (H1), (H2) be satisfied. Then the PBVP (1.1) possesses extremal periodic solutions, i.e., the greatest solution $u^{*}$ and the least solution $u_{*}$, within the sector $[\underline{u}, \bar{u}]$ formed by the lower and upper solution $\underline{u}$ and $\bar{u}$, respectively.

In the proof of Theorem 2.1 which will be given in section 4 we focus on the existence of the greatest solution only, since the existence of the least solution can be shown analogously. Also all preliminary results aim at this goal.

\section{PRELIMINARIES}

Throughout this section we shall assume that the hypotheses (A1)-(A3) and (H1), (H2) are satisfied.

Lemma 3.1. Let $u_{1}, u_{2} \in \mathcal{W}$ be any lower solutions of PBVP (1.1) with $u_{1}, u_{2} \in[\underline{u}, \bar{u}]$, where $\underline{u}$ and $\bar{u}$ are the given lower and upper solutions, respectively, according to hypothesis (H1). Then there exists a solution $u$ of the PBVP (1.1) satisfying $u_{0}:=\max \left(u_{1}, u_{2}\right) \leq u \leq \bar{u}$.

Proof. a) Existence result for an auxiliary problem

We define truncation operators $T_{i}, i=0,1,2$ that are related with the functions $u_{0}=\max \left(u_{1}, u_{2}\right), u_{1}, u_{2}$, respectively, by

$$
T_{i} u(x, t)=\left\{\begin{array}{rll}
\bar{u}(x, t) & \text { if } \quad & u(x, t)>\bar{u}(x, t), \\
u(x, t) & \text { if } \quad & u_{i}(x, t) \leq u(x, t) \leq \bar{u}(x, t), \\
u_{i}(x, t) & \text { if } & u(x, t)<u_{i}(x, t) .
\end{array}\right.
$$

It is well known that these operators $T_{i}: \mathcal{V} \mapsto \mathcal{V}$ are bounded and continuous (cf. [6]) which implies by (H2) that the composed operators $F \circ T_{i}: \mathcal{V} \mapsto$ 
$L^{q}(Q)$ are bounded and continuous as well. Furthermore, we introduce the following cut off function $b: Q \times \mathbb{R} \mapsto \mathbb{R}$ by

$$
b(x, t, s)=\left\{\begin{array}{rll}
(s-\bar{u}(x, t))^{p-1} & \text { if } \quad & s>\bar{u}(x, t), \\
0 & \text { if } \quad & u_{0}(x, t) \leq s \leq \bar{u}(x, t), \\
-\left(u_{0}(x, t)-s\right)^{p-1} & \text { if } \quad & s<u_{0}(x, t) .
\end{array}\right.
$$

Then one readily verifies that $b$ is a Carathéodory function satisfying a growth condition of the form

$$
|b(x, t, s)| \leq k_{3}(x, t)+c_{2}|s|^{p-1}
$$

for some positive constant $c_{2}$ and some function $k_{3} \in L^{q}(Q)$, and an estimate of the form

$$
\int_{Q} b(x, t, u(x, t)) u(x, t) d x d t \geq c_{3}\|u\|_{L^{p}(Q)}^{p}-c_{4}
$$

is valid for some positive constant $c_{3}, c_{4}$.

By (3.1) it follows that the Nemytskij operator $B$ associated with the function $b$ is bounded and continuous from $L^{p}(Q)$ into $L^{q}(Q)$.

Our approach is heavily based on existence and comparison results of the following auxiliary PBVP

$$
\left.\begin{array}{r}
\frac{\partial u}{\partial t}+A u+\gamma B u=F \circ T_{0} u+\sum_{i=1}^{2}\left|F \circ T_{i} u-F \circ T_{0} u\right| \text { in } Q, \\
u(x, 0)=u(x, \tau) \text { in } \Omega \text { and } u=0 \text { on } \Gamma .
\end{array}\right\}
$$

Let $L=\partial / \partial t$ and its domain $D(L) \subset \mathcal{V}_{0}$ given by

$$
D(L)=\left\{u \in \mathcal{W}_{0} \mid u(\cdot, 0)=u(\cdot, \tau) \text { in } \Omega\right\}
$$

where $L: D(L) \subset \mathcal{V}_{0} \mapsto \mathcal{V}_{0}^{*}$ is defined by

$$
\langle L u, \varphi\rangle=\int_{0}^{\tau}<\frac{\partial u}{\partial t}(t), \varphi(t)>d t \quad \text { for all } \varphi \in \mathcal{V}_{0}
$$

where $\langle\cdot, \cdot\rangle$ denotes the duality pairing between $W^{-1, q}(\Omega)$ and $W_{0}^{1, p}(\Omega)$. The linear operator $L: D(L) \subset \mathcal{V}_{0} \mapsto \mathcal{V}_{0}^{*}$ can be shown to be closed, densely defined and maximal monotone, cf. [12, Chapter 32]. Let us denote

$$
P u:=F \circ T_{0} u+\sum_{i=1}^{2}\left|F \circ T_{i} u-F \circ T_{0} u\right|,
$$


then by (H2) $P: \mathcal{V}_{0} \mapsto \mathcal{V}_{0}^{*}$ is bounded and continuous and for any $\varepsilon>0$ an estimate of the form

$$
|\langle P u, u\rangle| \leq \varepsilon\|\nabla u\|_{L^{p}(Q)}^{p}+C(\varepsilon)\|u\|_{L^{p}(Q)}^{p}+c\|u\|_{L^{p}(Q)}
$$

holds. By hypotheses (A1) and (A2) for any $\eta>0$ we have an estimate below

$$
\langle A u, u\rangle \geq \mu\|\nabla u\|_{L^{p}(Q)}^{p}-\eta\|\nabla u\|_{L^{p}(Q)}^{p}-C(\eta)\left(\left\|k_{0}\right\|_{L^{q}(Q)}^{q}+\|u\|_{L^{p}(Q)}^{p}\right) .
$$

The PBVP (3.3) may be given the form:

Find $u \in D(L) \subset \mathcal{V}_{0}$ such that

$$
(L+A-P+\gamma B) u=0,
$$

where the constant $\gamma>0$ will be specified later. The Leray-Lions conditions (A1) and (A2) along with the properties of the operators $B$ and $P$ imply that the operator $\mathcal{A}$ given by

$$
\mathcal{A}:=A-P+\gamma B
$$

gives rise to a continuous and bounded mapping from $\mathcal{V}_{0}$ into its dual $\mathcal{V}_{0}^{*}$ Moreover, $\mathcal{A}: \mathcal{V}_{0} \mapsto \mathcal{V}_{0}^{*}$ is pseudomonotone with respect to the graph norm topology of $D(L)$ which means that for any sequence $\left(u_{n}\right)$ in $D(L)$ with $u_{n} \rightarrow u$ in $\mathcal{V}_{0}, L u_{n} \rightarrow L u$ in $\mathcal{V}_{0}^{*}$ and $\lim \sup \left\langle\mathcal{A} u_{n}, u_{n}-u\right\rangle \leq 0$ it follows $\mathcal{A} u_{n} \rightarrow \mathcal{A} u$ in $\mathcal{V}_{0}^{*}$ and $\left\langle\mathcal{A} u_{n}, u_{n}\right\rangle \rightarrow\langle\mathcal{A} u, u\rangle$, cf. e.g. [2]. Applying [2, Theorem 5] (see also [1, Theorem 1]) the mapping $L+\mathcal{A}: D(L) \mapsto \mathcal{V}_{0}^{*}$ is surjective provided that $\mathcal{A}: \mathcal{V}_{0} \mapsto \mathcal{V}_{0}^{*}$ is coercive, i.e.,

$$
\frac{\langle\mathcal{A} u, u\rangle}{\|u\|_{\mathcal{V}_{0}}} \rightarrow \infty \quad \text { as }\|u\|_{\mathcal{V}_{0}} \rightarrow \infty
$$

The coercivity of $\mathcal{A}$ follows from (3.2), (3.4) and (3.5) for $\varepsilon$ and $\eta$ sufficiently small such that $\mu>\varepsilon+\eta$ and by choosing $\gamma$ sufficiently large. Hence [2, Theorem 5] implies the existence of at least one solution of the auxiliary PBVP (3.3).

b) Comparison

Here we show that any solution $u$ of the auxiliary problem (3.3) satisfies $\bar{u} \geq u \geq u_{i}$ for $i=1,2$ which implies that also $\bar{u} \geq u \geq u_{0}$ is fulfilled. Hence, for any solution of (3.3) it follows $T_{i} u=u$ which in turn implies that $P u=F u$ and $B u=0$ and thus $u$ must be a solution of the original problem (1.1) satisfying $u_{0} \leq u \leq \bar{u}$ which proves Lemma 3.1. In what follows we show that any solution $u$ of (3.3) satisfies $u \geq u_{k}$ for $k \in\{1,2\}$.

Since $u$ is a solution of (3.3) it satisfies

$$
L u+A u+\gamma B u=P u, \quad u(\cdot, 0)=u(\cdot, \tau)
$$


and the lower solution $u_{k}$ satisfies the inequality (with respect to the dual order cone)

$$
\frac{\partial u_{k}}{\partial t}+A u_{k} \leq F u_{k}
$$

as well as

$$
u_{k}(\cdot, 0) \leq u_{k}(\cdot, \tau) \text { and } u_{k} \leq 0 \text { on } \Gamma
$$

By (A3) for any $\varepsilon>0$ there exists a $\delta(\varepsilon) \in(0, \varepsilon)$ such that

$$
\int_{\delta(\varepsilon)}^{\varepsilon} \frac{d r}{\omega^{q}(r)}=1
$$

We introduce the function $h_{\varepsilon}: \mathbb{R} \mapsto \mathbb{R}_{+}$defined by (cf. [5])

$$
h_{\varepsilon}(t)=\left\{\begin{array}{rlll}
0 & \text { if } & t<\delta(\varepsilon), \\
\int_{\delta(\varepsilon)}^{t} \frac{d r}{\omega^{q}(r)} & \text { if } & \delta(\varepsilon) \leq t \leq \varepsilon, \\
1 & \text { if } & t>\varepsilon .
\end{array}\right.
$$

For any $\varepsilon>0$ the function $h_{\varepsilon}$ is Lipschitz continuous, nondecreasing and satisfies

$$
h_{\varepsilon}(t) \rightarrow \chi_{\{t>0\}} \quad \text { as } \quad \varepsilon \rightarrow 0,
$$

where $\chi_{\{t>0\}}$ denotes the characteristic function of the set $\{t>0\}$, as well as

$$
0 \leq h_{\varepsilon}^{\prime}(t)=\left\{\begin{aligned}
\frac{1}{\omega^{q}(t)} & \text { for } \delta(\varepsilon) \leq t \leq \varepsilon \\
0 & \text { otherwise }
\end{aligned}\right.
$$

The difference $u_{k}-u$ satisfies the inequalities

$$
\left(u_{k}-u\right)(\cdot, 0) \leq\left(u_{k}-u\right)(\cdot, \tau) \quad \text { and } u_{k}-u \leq 0 \quad \text { on } \Gamma \text {. }
$$

Subtracting (3.8) from (3.9) and taking advantage of the special nonnegative test function $\varphi$ in the form $\varphi=h_{\varepsilon}\left(u_{k}-u\right) \in \mathcal{V}_{0}$ we get

$$
\begin{aligned}
& \left\langle\frac{\partial\left(u_{k}-u\right)}{\partial t}, h_{\varepsilon}\left(u_{k}-u\right)\right\rangle+\left\langle A u_{k}-A u, h_{\varepsilon}\left(u_{k}-u\right)\right\rangle \\
& \leq \int_{Q}\left(F u_{k}-P u+\gamma B u\right) h_{\varepsilon}\left(u_{k}-u\right) d x d t
\end{aligned}
$$


Let $H_{\varepsilon}$ be a primitive of the nonnegative function $h_{\varepsilon}$ then by (3.11) the first term on the left-hand side of (3.12) yields the estimate (cf. e.g. [5])

$$
\begin{aligned}
& \left\langle\frac{\partial\left(u_{k}-u\right)}{\partial t}, h_{\varepsilon}\left(u_{k}-u\right)\right\rangle \\
& =\int_{\Omega} H_{\varepsilon}\left(u_{k}-u\right)(x, \tau) d x-\int_{\Omega} H_{\varepsilon}\left(u_{k}-u\right)(x, 0) d x \geq 0
\end{aligned}
$$

while the second term on the left-hand side of (3.12) can be estimated below in the following way using (A2) and (A3)

$$
\begin{aligned}
& \left\langle A u_{k}-A u, h_{\varepsilon}\left(u_{k}-u\right)\right\rangle \\
& =\sum_{i=1}^{N} \int_{Q}\left(a_{i}\left(x, t, u_{k}, \nabla u_{k}\right)-a_{i}(x, t, u, \nabla u)\right) \frac{\partial}{\partial x_{i}} h_{\varepsilon}\left(u_{k}-u\right) d x d t \\
& \geq \mu \int_{Q}\left|\nabla\left(u_{k}-u\right)\right|^{p} h_{\varepsilon}^{\prime}\left(u_{k}-u\right) d x d t \\
& -N \int_{Q}\left[\left|k_{1}\right|+\left|u_{k}\right|^{p-1}+|u|^{p-1}+|\nabla u|^{p-1}\right] \omega\left(\left|u_{k}-u\right|\right) \times \\
& \times h_{\varepsilon}^{\prime}\left(u_{k}-u\right)\left|\nabla\left(u_{k}-u\right)\right| d x d t \\
& \geq \frac{\mu}{2} \int_{Q}\left|\nabla\left(u_{k}-u\right)\right|^{p} h_{\varepsilon}^{\prime}\left(u_{k}-u\right) d x d t \\
& -c(\mu) \int_{Q} g^{q} \omega^{q}\left(\left|u_{k}-u\right|\right) h_{\varepsilon}^{\prime}\left(u_{k}-u\right) d x d t,
\end{aligned}
$$

where $g=\left|k_{1}\right|+\left|u_{k}\right|^{p-1}+|u|^{p-1}+|\nabla u|^{p-1} \in L^{q}(Q)$. By the definition of the function $h_{\varepsilon}$ we obtain from (3.14)

$$
\left\langle A u_{k}-A u, h_{\varepsilon}\left(u_{k}-u\right)\right\rangle \geq-c(\mu) \int_{\left\{\delta(\varepsilon)<u_{k}-u<\varepsilon\right\}} g^{q} d x d t
$$

where the term on the right-hand side of (3.15) tends to zero as $\varepsilon \rightarrow 0$.

By Lebesgue dominated convergence theorem the right-hand side of (3.12) converges to

$$
\begin{aligned}
\lim _{\varepsilon \rightarrow 0} \int_{Q}\left(F u_{k}-P u+\gamma B u\right) h_{\varepsilon}\left(u_{k}-u\right) d x d t \\
=\int_{Q}\left(F u_{k}-F \circ T_{0} u-\sum_{i=1}^{2}\left|F \circ T_{i} u-F \circ T_{0} u\right|\right. \\
\quad+\gamma B u) \chi_{\left\{u_{k}-u>0\right\}} d x d t \\
\leq \gamma \int_{Q} B u \chi_{\left\{u_{k}-u>0\right\}} d x d t=-\gamma \int_{\left\{u_{k}-u>0\right\}}\left(u_{0}-u\right)^{p-1} d x d t \\
\leq-\gamma \int_{Q}\left[\left(u_{k}-u\right)^{+}\right]^{p-1} d x d t \leq 0
\end{aligned}
$$


where $v^{+}=\max (v, 0)$. Hence, from (3.13), (3.15) and (3.16) we get as $\varepsilon \rightarrow 0$

$$
0 \leq \int_{Q}\left[\left(u_{k}-u\right)^{+}\right]^{p-1} d x d t \leq 0,
$$

which proves that $u_{k} \leq u$ for $k=1,2$ and thus $u_{0} \leq u$. In the same way one can show that any solution $\mathrm{u}$ of the auxiliary problem satisfies $u \leq \bar{u}$. This completes the proof of the lemma.

Corollary 3.1. Let $\mathcal{S}$ denote the solution set of the PBVP (1.1) enclosed by the upper and lower solution $\bar{u}$ and $\underline{u}$, respectively, i.e.,

$$
\mathcal{S}=\left\{u \in \mathcal{W}_{0} \mid u \in[\underline{u}, \bar{u}] \text { and } u \text { is a solution of the PBVP (1.1) }\right\} .
$$

Then this set $\mathcal{S}$ is directed which means that whenever $u_{1}, u_{2} \in \mathcal{S}$ there exists an element $u_{3} \in \mathcal{S}$ such that $u_{1} \leq u_{3}$ and $u_{2} \leq u_{3}$.

Proof. Since $u_{1}$ and $u_{2}$ are in particular lower solutions of the PBVP (1.1), by Lemma 3.1 there exists a solution $u_{3}$ within the order interval $\left[\max \left(u_{1}\right.\right.$, $\left.\left.u_{2}\right), \bar{u}\right]$ which proves the assertion of the corollary.

The following result has been proved in [3, Lemma 3.1]

Lemma 3.2. A norm-bounded and well-ordered chain $\mathcal{C}$ of $\mathcal{W}_{0}$ contains an increasing sequence which converges to $\sup \mathcal{C}$ weakly in $\mathcal{W}_{0}$ and strongly in $L^{p}(Q)$.

\section{PROOF OF THEOREM 2.1}

The proof of Theorem 2.1 will be given for the existence of the greatest solution $u^{*}$ only, since the existence of the smallest solution $u_{*}$ can shown by obvious dual reasoning.

First we show that the solution set $\mathcal{S}$ is uniformly bounded in $\mathcal{W}_{0}$, i.e.,

$$
\|u\|_{\mathcal{W}_{0}} \leq c \quad \text { for all } u \in \mathcal{S} .
$$

To this end let $u \in \mathcal{S}$ be arbitrarily given and take as special test function this solution. Then we get

$$
\langle L u, u\rangle+\langle A u-F u, u\rangle=0,
$$

where $u(\cdot, 0)=u(\cdot, \tau)$. The periodicity condition yields $\langle L u, u\rangle=0$. Since all solutions from $\mathcal{S}$ are uniformly $L^{p}(Q)$-bounded we obtain from

$$
\langle A u-F u, u\rangle=0,
$$

and by means of (3.5) and the estimate of the form (for any $\varepsilon>0$ )

$$
|\langle F u, u\rangle| \leq \varepsilon\|\nabla u\|_{L^{p}(Q)}^{p}+C(\varepsilon)\|u\|_{L^{p}(Q)}^{p}+c\|u\|_{L^{p}(Q)}
$$


by choosing the constants $\varepsilon$ and $\eta$ sufficiently small a uniform bound for the gradients which implies

$$
\|u\|_{\mathcal{V}_{0}} \leq c \quad \text { for all } u \in \mathcal{S}
$$

Finally, by means of (A1), (H2) and the uniform bound (4.3) we get

$$
|\langle L u, \varphi\rangle| \leq|\langle A u, \varphi\rangle|+|\langle F u, \varphi\rangle| \leq c \quad \text { for all } \varphi \in \mathcal{V}_{0}:\|\varphi\|_{\mathcal{V}_{0}} \leq 1
$$

which implies $\|L u\|_{\mathcal{V}_{0}^{*}} \leq c$ and thus the uniform estimate (4.1) holds.

Next we shall show that Zorn's lemma may be applied to the set $\mathcal{S}$. To this end let $\mathcal{C}$ be any well-ordered chain from $\mathcal{S}$. By (4.1) this chain is normbounded in $\mathcal{W}_{0}$ and hence from Lemma 3.2 there exists a nondecreasing sequence $\left(u_{n}\right)$ converging to some function $w=\sup \mathcal{C} \in \mathcal{W}_{0}$ weakly in $\mathcal{W}_{0}$ and strongly in $L^{p}(Q)$. Since $u_{n} \in D(L)$ and $D(L)$ is closed with respect to the norm in $\mathcal{W}_{0}$ and convex, it follows that the limit $w \in D(L)$. Furthermore, we have

$$
\begin{aligned}
\left\langle(A-F) u_{n}, u_{n}-w\right\rangle & =-\left\langle L u_{n}, u_{n}-w\right\rangle \\
& =-\left\langle L\left(u_{n}-w\right), u_{n}-w\right\rangle-\left\langle L w, u_{n}-w\right\rangle \rightarrow 0
\end{aligned}
$$

as $n \rightarrow \infty$, and by the pseudomonotonicity of the operator $A-F: \mathcal{V}_{0} \mapsto \mathcal{V}_{0}^{*}$ with respect to $D(L)$ it follows that (cf. [1])

$$
(A-F) u_{n} \rightarrow(A-F) w \text { in } \mathcal{V}_{0}^{*} \text { and }\left\langle(A-F) u_{n}, u_{n}\right\rangle \rightarrow\langle(A-F) w, w\rangle
$$

The convergence properties of the sequence $\left(u_{n}\right)$ and (4.4) allow to pass to the limit as $n \rightarrow \infty$ in the equation

$$
\left\langle(L+A-F) u_{n}, \varphi\right\rangle=0 \quad \text { for all } \varphi \in \mathcal{V}_{0},
$$

which proves that the limit $w=\sup \mathcal{C}$ is in $\mathcal{S}$. Thus we have shown that any well-ordered chain $\mathcal{C}$ of $\mathcal{S}$ possesses an upper bound in $\mathcal{S}$. By applying Zorn's lemma the existence of a maximal element $u_{m} \in \mathcal{S}$ (with respect to the underlying partial ordering) can be deduced. By Corollary 3.1 the set $\mathcal{S}$ is directed which implies that the maximal element $u_{m}$ is uniquely defined and must be the greatest one.

This completes the proof of Theorem 2.1.

4.1. Special case. Assume instead of hypothesis (A2) the weaker ones $\left(\mathrm{A} 2_{1}\right)$ and $\left(\mathrm{A} 2_{2}\right)$, and assume instead of (2.1) the more restrictive condition (2.2). We are going to justify the assertion given in Remark 2.1.

The only place where the modulus of continuity comes into picture and where the interplay with the monotonicity condition appears is in the part b) of the proof of Lemma 3.1 that deals with the comparison of lower solutions of the PBVP (1.1) and a solution of the auxiliary PBVP (3.3). The crucial 
step is to show that under the hypotheses $\left(\mathrm{A} 22_{1}\right)$ and $\left(\mathrm{A} 2_{2}\right)$ and $(2.2)$ the estimate (3.15) holds true. In this case by (2.2) for any $\varepsilon>0$ there exists a $\delta(\varepsilon) \in(0, \varepsilon)$ such that

$$
\int_{\delta(\varepsilon)}^{\varepsilon} \frac{d r}{\omega(r)}=1
$$

Now we introduce the function $h_{\varepsilon}: \mathbb{R} \mapsto \mathbb{R}_{+}$given by

$$
h_{\varepsilon}(t)=\left\{\begin{array}{rll}
0 & \text { if } & t<\delta(\varepsilon), \\
\int_{\delta(\varepsilon)}^{t} \frac{d r}{\omega(r)} & \text { if } & \delta(\varepsilon) \leq t \leq \varepsilon, \\
1 & \text { if } & t>\varepsilon .
\end{array}\right.
$$

Again we have that for any $\varepsilon>0$ the function $h_{\varepsilon}$ is Lipschitz continuous, nondecreasing and satisfies

$$
h_{\varepsilon}(t) \rightarrow \chi_{\{t>0\}} \quad \text { as } \quad \varepsilon \rightarrow 0,
$$

where $\chi_{\{t>0\}}$ denotes the characteristic function of the set $\{t>0\}$, as well as

$$
0 \leq h_{\varepsilon}^{\prime}(t)=\left\{\begin{aligned}
\frac{1}{\omega(t)} & \text { for } \delta(\varepsilon) \leq t \leq \varepsilon \\
0 & \text { otherwise }
\end{aligned}\right.
$$

In order to show that an estimate similar to that of (3.15) is true also under the new assumptions we estimate the term $\left\langle A u_{k}-A u, h_{\varepsilon}\left(u_{k}-u\right)\right\rangle$ below where $h_{\varepsilon}$ is given by (4.4).

$$
\begin{aligned}
& \left\langle A u_{k}-A u, h_{\varepsilon}\left(u_{k}-u\right)\right\rangle \\
& =\sum_{i=1}^{N} \int_{Q}\left(a_{i}\left(x, t, u_{k}, \nabla u_{k}\right)-a_{i}(x, t, u, \nabla u)\right) \frac{\partial}{\partial x_{i}} h_{\varepsilon}\left(u_{k}-u\right) d x d t \\
& \geq \sum_{i=1}^{N} \int_{Q}\left(a_{i}\left(x, t, u_{k}, \nabla u_{k}\right)-a_{i}\left(x, t, u_{k}, \nabla u\right)\right) \\
& \quad \times \frac{\partial\left(u_{k}-u\right)}{\partial x_{i}} h_{\varepsilon}^{\prime}\left(u_{k}-u\right) d x d t \\
& \quad-N \int_{Q}\left[\left|k_{1}\right|+\left|u_{k}\right|^{p-1}+|u|^{p-1}+|\nabla u|^{p-1}\right] \omega\left(\left|u_{k}-u\right|\right) \times \\
& \quad \times h_{\varepsilon}^{\prime}\left(u_{k}-u\right)\left|\nabla\left(u_{k}-u\right)\right| d x d t \\
& \geq \\
& \quad-N \int_{\left\{\delta(\varepsilon)<u_{k}-u<\varepsilon\right\}} g\left|\nabla\left(u_{k}-u\right)\right| d x d t
\end{aligned}
$$


where $g=\left|k_{1}\right|+\left|u_{k}\right|^{p-1}+|u|^{p-1}+|\nabla u|^{p-1} \in L^{q}(Q)$. Since the term on the right-hand side of (4.5) tends to zero as $\varepsilon \rightarrow 0$ we have an estimate of the form (3.15) and the comparison follows from here the same way as in part b) of the proof of Lemma 3.1.

Acknowledgment. I am very grateful to Professor V. Mustonen for discussions on the subject of this paper during my stay at the University of Oulu.

\section{REFERENCES}

1. J. Berkovits and V. Mustonen, Monotonicity methods for nonlinear evolution equations, Nonlinear Anal. 27 (1996), 1397-1405.

2. J. Berkovits and V. Mustonen, Topological degree for perturbations of linear maximal monotone mappings and applications to a class of parabolic problems, Rend. Mat. Appl. (7) 12 (1992), 597-621.

3. S. Carl, On the existence of extremal weak solutions for a class of quasilinear parabolic problems, Differential Integral Equations 6 (1993), 1493-1505.

4. S. Carl, An envelope theorem for weak solutions of quasilinear elliptic boundary value problems, World Congress of Nonlinear Analysts '92 (V. Lakshmikantham ed.), Walter de Gruyter, Berlin-New York, 1996, pp. 869-877..

5. M. Chipot and J. F. Rodrigues, Comparison and stability of solutions to a class of quasilinear parabolic problems, Proc. Roy. Soc. Edinburgh Sect. A 110 (1988), 275-285.

6. J. Deuel and P. Hess, A criterion for the existence of solutions of non-linear elliptic boundary value problems, Proc. Roy. Soc. Edinburgh Sect. A 74 (1975), 49-54.

7. Nonlinear parabolic boundary value problems with upper and lower solutions, Israel J. Math. 29 (1978), 92-104.

8. N. Grenon, Existence result for some quasilinear parabolic problems, Ann. Mat. Pura Appl. (4) 165 (1993), 281-313.

9. __ Asymptotic behaviour for some quasilinear parabolic equations, Nonlinear Anal. 20 (1993), 755-766.

10. J.-L. Lions, Quelques Méthodes de Résolution des Problémes aux Limites Nonlinéaires, Dunod Gauthier-Villars, Paris, 1969.

11. J. P. Puel, Some results on quasi-linear elliptic equations, Pitman Res. Notes Math. Ser. 208 (1989), 306-318.

12. E. Zeidler, Nonlinear Functional Analysis and its Applications, II B, Springer, Berlin, 1990.

Martin-Luther-Universität Halle-Wittenberg

FACHBEREICH MATHEMATIK UND INFORMATIK

INSTITUT FÜR ANALYSIS

D-06099 HaLle, GERMANY

E-mail address: carl@condor.mathematik.uni-halle.de 


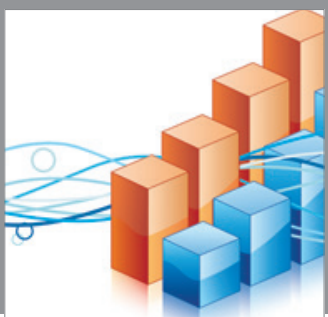

Advances in

Operations Research

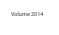

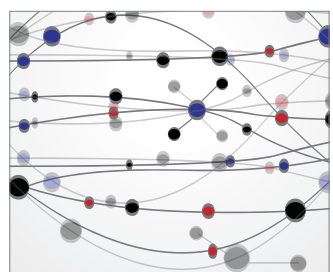

\section{The Scientific} World Journal
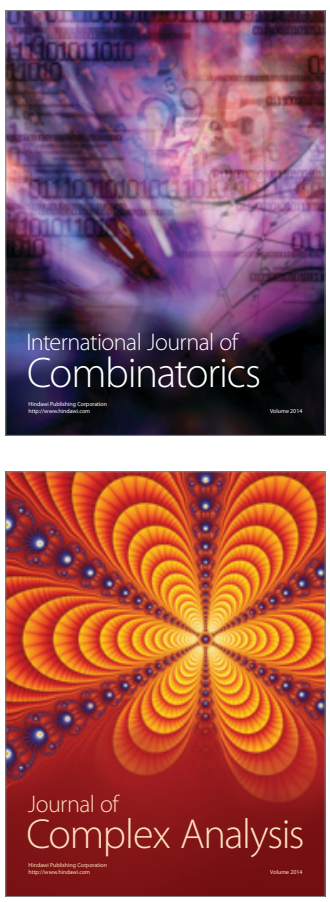

International Journal of

Mathematics and

Mathematical

Sciences
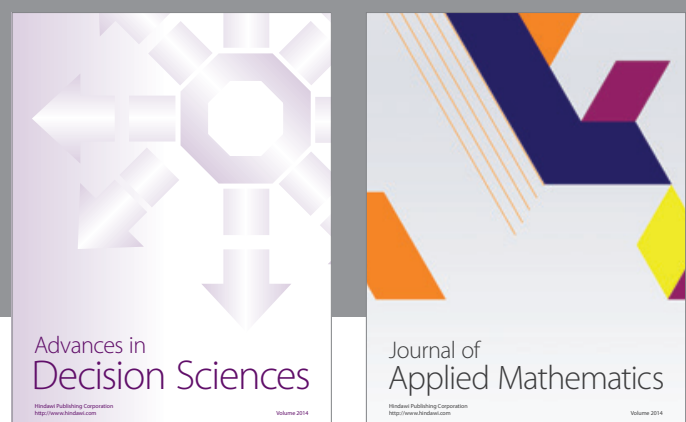

Journal of

Applied Mathematics
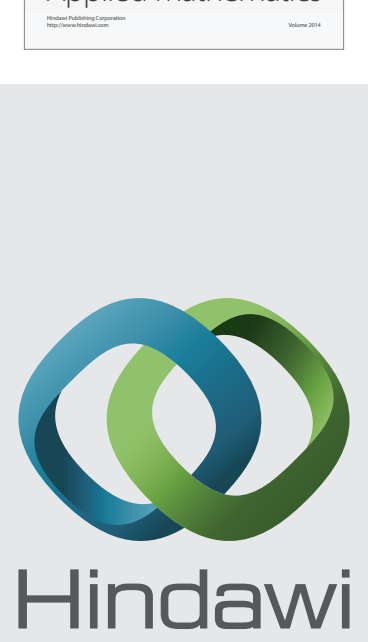

Submit your manuscripts at http://www.hindawi.com
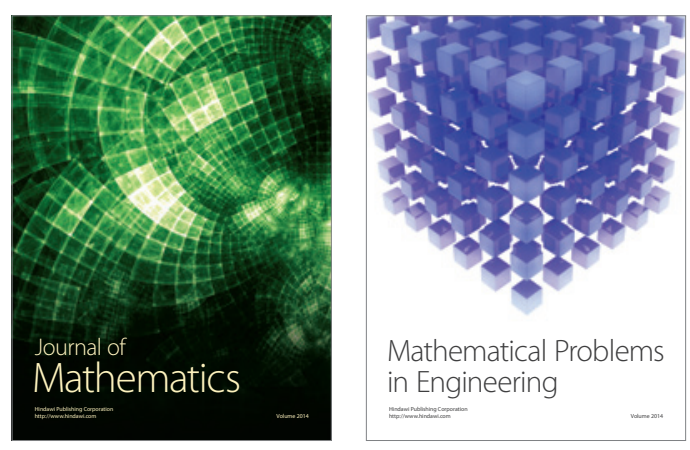

Mathematical Problems in Engineering
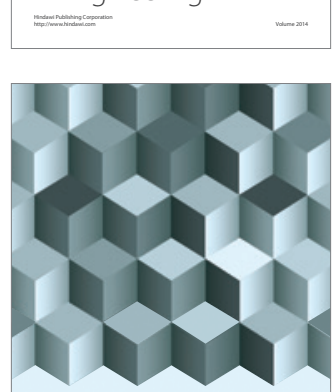

Journal of

Function Spaces
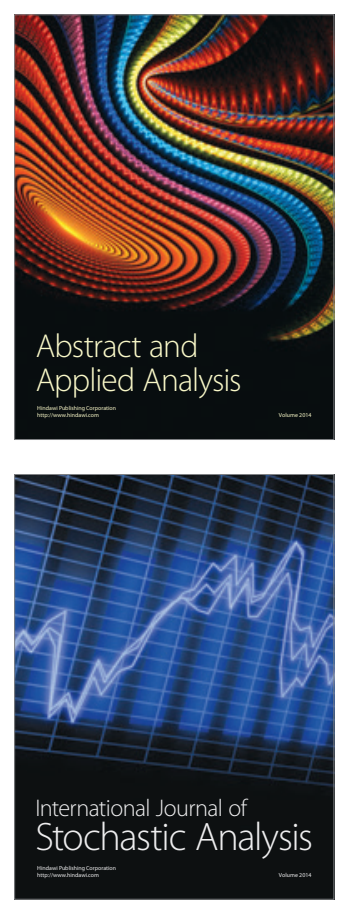

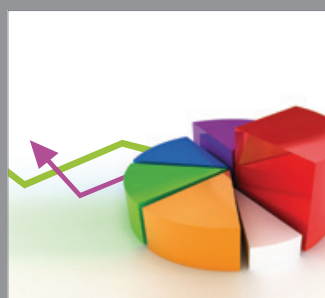

ournal of

Probability and Statistics

Promensencen
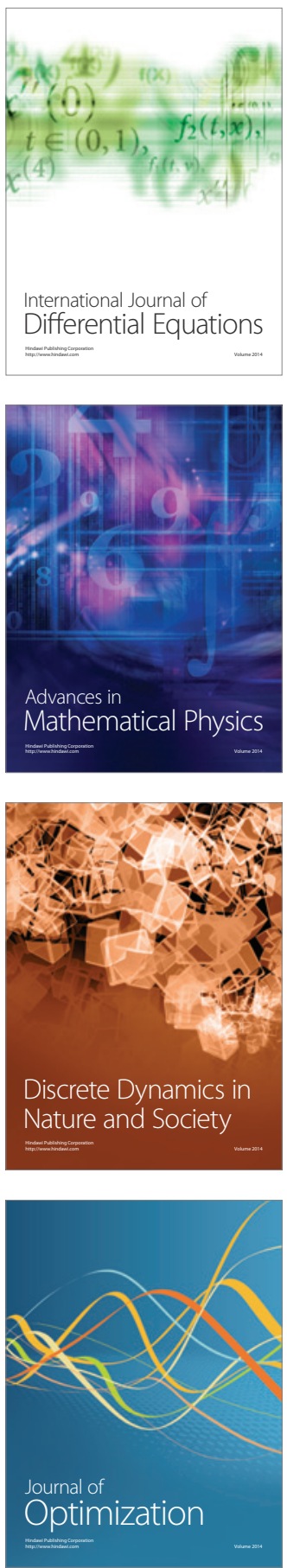